\title{
MULTIGOAL OUTPUT REGULATION VIA SUPERVISORY CONTROL: APPLICATION TO STABILIZATION OF A UNICYCLE
}

\author{
Denis Efimov Antonio Loría Elena Panteley
}

\begin{abstract}
We consider the problem of robust output regulation for nonlinear systems in the following sense: given a system with input disturbances, we are interested in steering one output to zero while tolerating an steady-state error on the second output. In other words, we are interested in inputoutput (asymptotic) stability for one output and practical (asymptotic) stability for the second. Upon the assumption that we dispose of two controllers each of which achieves separately a control objective (for either output) we propose a switching rule to commute between the two controllers appropriately i.e., conserving the input-output stability, to some extent. The problem studied is reminiscent of different concrete situations such as simultaneaous synchronization and tracking control of mechanical systems; in this article we revisit the problem of stabilizing a unicycle (regulation of the first output) towards a point while ensuring obstacle-collision avoidance (practical stabilization of the second output).
\end{abstract}

\section{INTRODUCTION}

There is a number of motivations to study switched control systems $-c f$. [1] and in particular, supervisory control $-c f$. . [2]. For instance, one may think of a situation in which a set of controllers, each acting on different subsets of the state space, achieves a different control goal for the same plant but no control alone achieves all objectives simultaneously e.g., perfect synchronization and tracking control under disturbances. Alternatively one may think of situations when different controllers may be used on the same state subspace, for the same plant, with the same goal but each achieving different performances (transient overshoots, speed of convergence, etc). As is wellknown stability of each independently-controlled system does not imply, in general, that the switched controlled system remains stable, let alone, that performance is improved or even conserved. For the case of uniting two controls, one "local" and another "global", the problem has different solutions: dynamic and static time invariant continuous controllers $-c f$. [3], [4]; supervisory control -cf. [5], [6], [7], [8], [9], [10]. Stability theory for switched systems is particularly useful to establish general results on how to "orchestrate" a collection of nonlinear systems, that is on the design of the switching rule $-c f$. [11], [12], [13]. Particular variants in this direction include dwell-time hysteresis supervisors -cf. e.g. , [?], [?]).

All of the latter deal with the case of either state or common output regulation however, in some practical cases it is required to stabilize the system under some restrictions on admissible values of a second output. An example of such a situation is analyzed in

D. Efimov is with the Dept. of Electr. Engg and Comp. Sc., B28 Universite de Liège, Belgium; E. Panteley and A. Loría are with with C.N.R.S. at Laboratoire des Signaux et Systèmes, SUPELEC, 3, Rue Joliot Curie, 91192 Gif s/Yvette, France. E-mail: loriallss.supelec.fr.
[?] in the context of synchronization of robot manipulators: the goal is to achieve asymptotic trajectory tracking of a common desired reference for all robots under the restriction that mutual synchronization errors remain within admissible bounds established a priori. Another example is the stabilization with respect to one state variable under the constraint that other state variables remain in a pre-specified domain $-c f$. [14]; this is reminiscent of many situations in adaptive control systems without parameter convergence.

In this paper we present a supervisor for the case of two controls and two different outputs. The main result are stated in terms of and aim at establishing input-output stability as opposed to input-to-state stability. We illustrate the use of our main theorem by revisiting a stabilization problem of a unicycle: to steer it to a desired configuration while avoiding obstacles. in the scheme, one regulated output corresponds to the configuration error relative to a desired reference and a second output, inversely proportional to the distance of the unicycle to obstacles, is meant to be kept within pre-specified limits.

The rest of the paper is organized as follows. Section II contains some definitions, notations and auxiliary statements. The problem that we address is formulated in in Section III and solved in Section IV. In the latter we also present the case-study of the unicycle. Proofs are presented in Section ?? and we conclude with some remarks in Section V.

\section{PRELIMINARIES}

Consider systems

$$
\dot{\mathbf{x}}=\mathbf{f}_{i}(\mathbf{x}, \mathbf{d}), \quad \mathbf{y}=\mathbf{h}(\mathbf{x}), \quad i \in I,
$$

where $\mathbf{x} \in R^{n}, \mathbf{d} \in R^{m}$ is an external disturbance, $\mathbf{y} \in R^{p}$ is an output; $i$ is an index taking values in the countable set $I$. We assume that the functions $\mathbf{f}_{i}: R^{n+l} \rightarrow R^{n}$ and $\mathbf{h}: R^{n} \rightarrow R^{p}$ are continuous and locally Lipschitz, $\mathbf{d}: R_{+} \rightarrow R^{m}$ is Lebesgue measurable and locally essentially bounded i.e.,

$$
\|\mathbf{d}\|_{\left[t_{0}, t\right)}=e s s \sup \left\{|\mathbf{d}(t)|, t \in\left[t_{0}, t\right)\right\}<\infty
$$

where $|\cdot|$ denotes Euclidean norm. The set of functions such that $\|\mathbf{d}\|:=\|\mathbf{d}\|_{[0,+\infty)}<+\infty$ is denoted by $L_{\infty}^{m}$. We introduce norm operator $S: L_{\infty}^{m} \times R^{2} \rightarrow R_{+}$, with $S\left[0, t_{0}, t\right]=0$, for any $t \geq t_{0} \geq 0$. The set of functions such that $S[\mathbf{d}, 0,+\infty]<$ $+\infty$ is denoted by $M_{R^{m}}\left(M_{R^{m}} \subseteq L_{\infty}^{m}\right)$. Qualifying norms are $S\left[\mathbf{d}, t_{0}, t\right]=\|\mathbf{d}\|_{\left[t_{0}, t\right)}$ and

$$
S\left[\mathbf{d}, t_{0}, t\right]=\int_{t_{0}}^{t} \omega(|\mathbf{d}(\tau)|) d \tau, \quad \omega \in K .
$$


Let $i: R_{+} \rightarrow I$ be piecewise constant continuous from the right then, systems (1) define the switched system

$$
\dot{\mathbf{x}}=\mathbf{f}_{i(t)}(\mathbf{x}, \mathbf{d}), \quad \mathbf{y}=\mathbf{h}(\mathbf{x}),
$$

where the switching signal $i(t)$ is said to have an average dwelltime $0<\tau_{D}<+\infty$ if, between switches, for any time instants $t_{2} \geq t_{1} \geq 0$ and an integer number $1 \leq N_{0}<+\infty$ we have

$$
N_{\left[t_{1}, t_{2}\right)} \leq N_{0}+\frac{t_{2}-t_{1}}{\tau_{D}}
$$

where $N_{\left[t_{1}, t_{2}\right)}$ is the number of discontinuities of $i(t)-c f$. [11], [12], [15], [16]. If the interval between any two switches is not less than $\tau_{D}$ then $i$ has dwell-time and $N_{0}=1$. System (2) with $i(t)$ having average dwell-time or simple dwell-time undergoes a finite number of switches on any finite-time interval and its solution is continuous and locally defined. If for $i(t)$, all initial conditions $\mathbf{x}_{0} \in R^{n}$ and inputs $\mathbf{d} \in M_{R^{m}}$ the solutions $\mathbf{x}\left(t, \mathbf{x}_{0}, \mathbf{d}\right)$ (corresp. $\left.\mathbf{y}\left(t, \mathbf{x}_{0}, \mathbf{d}\right)=\mathbf{h}\left(\mathbf{x}\left(t, \mathbf{x}_{0}, \mathbf{d}\right)\right)\right)$ are defined for all $t \geq 0$ then the switched system is forward complete.

Definition $1-c f$. [17]. For fixed $i \in I$ the forward complete system (1) is state independent IOS (SIIOS) with respect to output $\mathbf{y}$ and input $\mathbf{d}$ with input-to-output norm operator $S$ if for all $\mathbf{x}_{0} \in R^{n}$ and $\mathbf{d} \in M_{R^{m}}$ there exist functions ${ }^{2} \beta_{i}^{\prime} \in K L$ and $\gamma_{i}^{\prime} \in K$ such that

$$
\left|\mathbf{y}\left(t, \mathbf{x}_{0}, \mathbf{d}\right)\right| \leq \beta_{i}^{\prime}\left(\left|\mathbf{h}\left(\mathbf{x}_{0}\right)\right|, t\right)+\gamma_{i}^{\prime}(S[\mathbf{d}, 0, t]), \quad t \geq 0 .
$$

The switched forward complete system (2) with $i: R_{+} \rightarrow I$ is SIIOS with respect to output $\mathbf{y}$ and input $\mathbf{d}$ with input norm operator $S$ if for all $\mathbf{x}_{0} \in R^{n}$ and $\mathbf{d} \in M_{R^{m}}$ there exist functions $\beta^{\prime} \in K L, \gamma^{\prime} \in K$ such that

$$
\left|\mathbf{y}\left(t, \mathbf{x}_{0}, \mathbf{d}\right)\right| \leq \beta^{\prime}\left(\left|\mathbf{h}\left(\mathbf{x}_{0}\right)\right|, t\right)+\gamma^{\prime}(S[\mathbf{d}, 0, t]), \quad t \geq 0 .
$$

\section{PROBLEM STATEMENT}

Consider the system

$$
\dot{\mathbf{x}}=\mathbf{f}(\mathbf{x}, \mathbf{u}, \mathbf{d}), \quad \mathbf{y}_{1}=\mathbf{h}_{1}(\mathbf{x}), \quad \mathbf{y}_{2}=\mathbf{h}_{2}(\mathbf{x}),
$$

where $\mathbf{x} \in R^{n}, \mathbf{u} \in R^{m}, \mathbf{d} \in M_{R^{k}} ; \mathbf{y}_{1} \in R^{p_{1}}, \mathbf{y}_{2} \in R^{p_{2}}$ are two outputs to be regulated, $\mathbf{h}_{1}, \mathbf{h}_{2}$ are continuous and $\mathbf{f}$ is locally Lipschitz continuous.

It is required to design a control $\mathbf{u}: R^{n} \rightarrow R^{m}$ providing robust output (property SIIOS) stabilization of the system with respect to the output $\mathbf{y}_{1}$ under the restriction of keeping $\mathbf{y}_{2}$ within a prescribed limit. Thus, the following estimates are required to hold for all initial conditions $\mathbf{x}_{0} \in R^{n}, \mathbf{d} \in M_{R^{k}}$ of the closed loop system and for all $t \geq t_{0} \geq 0$ :

$$
\begin{gathered}
\left|\mathbf{y}_{1}\left(t, \mathbf{x}_{0}, \mathbf{d}\right)\right| \leq \beta\left(\left|\mathbf{h}_{1}\left(\mathbf{x}_{0}\right)\right|, t-t_{0}\right)+\gamma\left(S\left[\mathbf{d}, t_{0}, t\right]\right), \\
\left|\mathbf{y}_{2}\left(t, \mathbf{x}_{0}, \mathbf{d}\right)\right| \leq \sigma_{1}\left(\max \left\{\Delta,\left|\mathbf{h}_{2}\left(\mathbf{x}_{0}\right)\right|\right\}\right)+\sigma_{2}\left(S\left[\mathbf{d}, t_{0}, t\right]\right),
\end{gathered}
$$

where $\Delta>0$ is given, $\beta \in K L, \gamma, \sigma_{1}, \sigma_{2} \in K$. Estimate (4) is a conventional SIIOS estimate, the estimate (5) implies that for initial conditions $\left|\mathbf{h}_{2}\left(\mathbf{x}_{0}\right)\right| \leq \Delta$ and without disturbances the output $\mathbf{y}_{2}$ is always smaller than $\sigma_{1}(\Delta)$; in the presence of disturbances $\mathbf{d} \in M_{R^{k}}$ deviations proportional to $\sigma_{2}\left(S\left[\mathbf{d}, t_{0}, t\right]\right)$

\footnotetext{
${ }^{2} \mathrm{~A}$ continuous function $\sigma: R_{+} \rightarrow R_{+}$belongs to class $K$ if it is strictly increasing and $\sigma(0)=0$; it belongs to class $K_{\infty}$ if it is also radially unbounded; continuous function $\beta: R_{+} \times R_{+} \rightarrow R_{+}$is of class $K L$, if $\beta(\cdot, t) \in K$ for any $t \in R_{+}$, and $\beta(s, \cdot)$ is strictly decreasing to zero for any $s \in R_{+}$.
}

are allowed. For $\left|\mathbf{h}_{2}\left(\mathbf{x}_{0}\right)\right|>\Delta$ trajectories should converge to the subset where $\left|\mathbf{h}_{2}(\mathbf{x})\right| \leq \Delta$.

The solvability of this problem imposes that the outputs to be controlled are such that the intersection of the set $\mathbf{y}_{1}=0$ with the set $\left|\mathbf{y}_{2}(\mathbf{x})\right| \leq \Delta$ is non-empty. Therefore, we shall assume (-cf. . Theorem 1 later) that there exist $\rho \in K$ and $\rho_{0}>0$ such that, for all $\mathrm{x} \in R^{n}$,

$$
\left|\mathbf{h}_{2}(\mathbf{x})\right| \leq \rho\left(\left|\mathbf{h}_{1}(\mathbf{x})\right|\right)+\rho_{0} .
$$

Also, note that Ineq. (4) may imply (5) only for $t \geq 0$ such that $\mathbf{h}_{1}(\mathbf{x}(t))$ is relatively small.

In the context of this paper we assume that independent output regulators are given then, we design a supervisor that orchestrates the switching between the regulators so as to guarantee that (4) and (5) hold.

Assumption 1. There exist controls $\mathbf{u}_{j}: R^{n} \rightarrow R^{m}, j \in\{1,2\}$ such that the system

$$
\dot{\mathbf{x}}=\mathbf{f}\left(\mathbf{x}, \mathbf{u}_{j}(\mathbf{x}), \mathbf{d}\right), \quad \mathbf{y}_{j}=\mathbf{h}_{j}(\mathbf{x}), \quad j \in\{1,2\}
$$

has continuous solutions $\mathbf{x}(t)$ defined for all $t \geq 0$, the functions $\mathbf{u}_{j}(\mathbf{x}(\cdot))$ are right-continuous and the system (7) is SIIOS with respect to outputs $\mathbf{y}_{j}$ and input $\mathbf{d}$ with functions $\beta_{j} \in K L, \gamma \in$ $K$. Moreover, there exist continuous functions $\omega_{j}: R_{+}^{4} \rightarrow R_{+}$ such that:

- $\omega_{1}$ is non-decreasing with respect to the first three arguments and increasing with respect to the last argument;

- $\omega_{2}$ is non-decreasing with respect to the first three arguments, non-increasing with respect to the last argument and $\omega_{2}(0, \cdot, 0, \cdot) \equiv 0$

- For all $\mathbf{x}_{0} \in R^{n}, \mathbf{d} \in M_{R^{m}}$ and all $t \geq t_{0} \geq 0$, the output trajectories of $\dot{\mathbf{x}}=\mathbf{f}\left(\mathbf{x}, \mathbf{u}_{1}(\mathbf{x}), \mathbf{d}\right)$, satisfy

$$
\left|\mathbf{y}_{2}\left(t, \mathbf{x}_{0}, \mathbf{d}\right)\right| \leq \omega_{1}\left(\left|\mathbf{h}_{1}\left(\mathbf{x}_{0}\right)\right|,\left|\mathbf{h}_{2}\left(\mathbf{x}_{0}\right)\right|, S\left[\mathbf{d}, t_{0}, t\right], t-t_{0}\right),
$$

and the output trajectories of $\dot{\mathbf{x}}=\mathbf{f}\left(\mathbf{x}, \mathbf{u}_{2}(\mathbf{x}), \mathbf{d}\right)$, satisfy

$$
\left|\mathbf{y}_{1}\left(t, \mathbf{x}_{0}, \mathbf{d}\right)\right| \leq \omega_{2}\left(\left|\mathbf{h}_{1}\left(\mathbf{x}_{0}\right)\right|,\left|\mathbf{h}_{2}\left(\mathbf{x}_{0}\right)\right|, S\left[\mathbf{d}, t_{0}, t\right], t-t_{0}\right) .
$$

In particular, we assume that control $\mathbf{u}_{1}$ guarantees that the system satisfies an estimate similar to (4) with known upper estimate for $\mathbf{y}_{2}$. Control $\mathbf{u}_{2}$ also implies that an estimate similar to (4) holds for the output $\mathbf{y}_{2}$ which is a stronger than (5).

\section{MAIN RESULTS}

By assumption we dispose of two controls which independently solve the output regulation problem for $\mathbf{y}_{1}$ and $\mathbf{y}_{2}$. We propose a supervisor to combine the controls $\mathbf{u}_{1}$ and $\mathbf{u}_{2}$ so as to ensure that (4), (5) hold for the closed-loop system. We assume that control $\mathbf{u}_{2}$ is immediately activated in the event that the output $\mathbf{y}_{2}$ reaches for the predefined level $\Delta ; u_{2}$ remains active for a minimum amount of time $\tau_{D}>0$ (dwell-time) units of time to ensure convergence of the system trajectories to the set $\left\{\left|\mathbf{y}_{2}(t)\right| \leq \Delta\right\}$. To ensure a better performance we switch on control $\mathbf{u}_{1}$ under the condition that $\left|\mathbf{y}_{2}(t)\right| \leq \delta$, where $\delta<\Delta$ is a design parameter. Then, the switched control takes the form

$$
\mathbf{U}(t)=\mathbf{u}_{i(t)}(\mathbf{x}(t)),
$$


where $i: R_{+} \rightarrow\{1,2\}$ is piecewise constant. The supervisor is defined as

$$
\begin{gathered}
t_{j+1}=\left\{\begin{array}{cc}
\underset{t \geq t_{j}}{\arg \inf } \mathbf{x}(t) \notin \mathbf{X}_{2} & \text { if } i\left(t_{j}\right)=1 ; \\
\underset{t \geq t_{j}+\tau_{D}}{\arg \operatorname{xin}} \mathbf{x}(t) \in \mathbf{X}_{1} & \text { if } i\left(t_{j}\right)=2,
\end{array}\right. \\
i\left(t_{j+1}\right)=\left\{\begin{array}{cc}
1, & \text { if } \mathbf{x}\left(t_{j+1}\right) \in \mathbf{X}_{1} ; \\
2, & \text { if } \mathbf{x}\left(t_{j+1}\right) \notin \mathbf{X}_{2},
\end{array}\right.
\end{gathered}
$$

$i(t)=i\left(t_{j}\right)$ for $t \in\left[t_{j}, t_{j+1}\right)$;

$$
\begin{gathered}
t_{0}=0, \quad i\left(t_{0}\right)= \begin{cases}1, & \text { if } \mathbf{x}\left(t_{0}\right) \in \mathrm{X}_{1} ; \\
2, & \text { otherwise, }\end{cases} \\
\mathbf{X}_{1}=\left\{\mathbf{x}:\left|\mathbf{h}_{2}(\mathbf{x})\right| \leq \delta\right\}, \quad \mathbf{X}_{2}=\left\{\mathbf{x}:\left|\mathbf{h}_{2}(\mathbf{x})\right|<\Delta\right\},
\end{gathered}
$$

where $t_{j}, j=0,1,2, \ldots$ are switching instants, $j$ is the number of the last switch; $\tau_{D}>0$ is the dwell-time constant and conditions on the threshold $\delta>0$ are specified below.

The control (8) equals to control $\mathbf{u}_{1}$ in set $\mathrm{X}_{1}$ (when amplitude of output $\mathbf{y}_{2}$ is smaller than some $\delta$ ) and to control $\mathbf{u}_{2}$ in the set $R^{n} \backslash \mathrm{X}_{2}$ (in this case norm of the output $\mathbf{y}_{2}$ is bigger than $\Delta$ and stabilization is required). The signal $i(t)$ takes constant values in the set $N=\mathbf{X}_{2} \backslash \mathbf{X}_{1}$ and plays a role of hysteresis in the system. Since $N$ may be non-compact dwell-time is used to avoid chattering however, dwell-time is used only for the control $\mathbf{u}_{2}$ which is in charge of the "stabilization" goal. If the trajectory leaves the set $\mathbf{X}_{2}$ then, without dwell-time, the control $\mathbf{u}_{2}$ is switched on to guarantee (5).

Theorem 1. Let Assumption 1 and the following hold.

- Let $D$ be a non-negative reals and define the set of (bounded) input disturbances $\Omega=\left\{\mathbf{d} \in M_{R^{k}}\right.$ : $S[\mathbf{d}, 0,+\infty] \leq D\}$

- let $R_{1} \geq 0$ and assume that Ineq. (6) holds then, define $X=\left\{\mathbf{x} \in R^{n}:\left|\mathbf{h}_{1}(\mathbf{x})\right| \leq R_{1}\right\}$ and $R_{2}=\rho\left(R_{1}\right)+\rho_{0} ;$

- consider $\omega_{1}$ and $\omega_{2}$ generated by Assumption 1 and assume that there exist $\delta$ and $\Delta$ such that

$$
\begin{gathered}
\Delta>\max \left\{\delta, \rho(\gamma(D))+\rho_{0}, \omega_{1}(s, \delta, D, 0)\right\} \quad \forall s \geq 0 \\
\delta>\gamma(D) ;
\end{gathered}
$$

- let $\beta_{1}, \beta_{2} \in \mathcal{K} \mathcal{L}$ be generated by Assumption $1, \tau_{D} \geq 0$ and $\lambda \in(0,1)$ be such that $T_{1}: R_{+} \rightarrow R_{+}$is a solution of $\Delta=\omega_{1}\left(s, \delta, D, T_{1}(s)\right)$ for all $s \geq 0$ and, defining

$$
\begin{aligned}
& R_{3}=\max \left\{\beta_{1}\left(R_{1}+\Lambda \gamma(D), 0\right)+\gamma(D),\right. \\
&\left.\omega_{2}\left(R_{1}+\Lambda \gamma(D), \max \left\{\Delta, R_{2}\right\}, \gamma(D), 0\right)\right\},
\end{aligned}
$$

we have, for all $s \in\left[0, R_{3}\right]$

$$
\begin{gathered}
\beta_{2}\left(\Delta, \tau_{D}\right)+\gamma(D)=\delta, \quad \beta_{1}\left(s, T_{1}(s)\right) \leq \lambda s, \\
\omega_{2}\left(s, \Delta, D, \tau_{D}\right) \leq s .
\end{gathered}
$$

Then, the system (3), (8), (9) is forward complete and for all $\mathbf{x}_{0} \in X, \mathbf{d} \in \Omega$ and all $t \geq t_{0} \geq 0$ we have

$$
\begin{gathered}
\left|\mathbf{y}_{1}(t)\right| \leq \max \left\{\omega _ { 2 } \left(\left|\mathbf{y}_{1}\left(t_{0}\right)\right|+\Lambda \gamma\left(S\left[\mathbf{d}, t_{0},+\infty\right]\right),\right.\right. \\
\left.\max \left\{\Delta,\left|\mathbf{y}_{2}\left(t_{0}\right)\right|\right\}, \gamma\left(S\left[\mathbf{d}, t_{0},+\infty\right]\right), 0\right), \\
\left.\beta_{1}\left(\left|\mathbf{y}_{1}\left(t_{0}\right)\right|+\Lambda \gamma\left(S\left[\mathbf{d}, t_{0},+\infty\right]\right), 0\right)+\gamma\left(S\left[\mathbf{d}, t_{0},+\infty\right]\right)\right\}
\end{gathered}
$$

$$
\begin{aligned}
& \lim _{t \rightarrow+\infty}\left|\mathbf{y}_{1}(t)\right| \leq \max \{ \\
& \omega_{2}\left(\Lambda \gamma\left(S\left[\mathbf{d}, t_{0},+\infty\right]\right), \max \left\{\Delta,\left|\mathbf{y}_{2}\left(t_{0}\right)\right|\right\}, \gamma\left(S\left[\mathbf{d}, t_{0},+\infty\right]\right), 0\right) \\
& \left.\quad \beta_{1}\left(\Lambda \gamma\left(S\left[\mathbf{d}, t_{0},+\infty\right]\right), 0\right)+\gamma\left(S\left[\mathbf{d}, t_{0},+\infty\right]\right)\right\}, \\
& \left|\mathbf{y}_{2}(t)\right| \leq \beta_{2}\left(\max \left\{\Delta,\left|\mathbf{y}_{2}\left(t_{0}\right)\right|\right\}, 0\right)+\gamma\left(S\left[\mathbf{d}, t_{0},+\infty\right]\right) .
\end{aligned}
$$

Remark 1. As it can be seen from the proof of Theorem 1 $c f$. Section ??, control $u_{1}$ should satisfy Assumption 1 only in the set $\mathbf{X}_{2}$ (since it is active only in this set). In this case control $u_{1}$ is "local" (set $\mathbf{X}_{2}$ can be non compact in general case) and control $\mathbf{u}_{2}$ is global.

The condition (10) of the theorem implies, that the output $\mathbf{y}_{1}$ of system (7) with $u_{1}$, decreases by a factor of $\lambda$ during the time $T_{1}$ while the output $\mathbf{y}_{2}$ does not exceed the threshold $\Delta$. On the other hand, from (10), while output $\mathbf{y}_{2}$ of (7) with control $u_{2}$ goes below the threshold $\delta$, the output $\mathbf{y}_{1}$ does not increase. In this case, consecutive switching between the controls $u_{1}$ and $u_{2}$ in (7) implies that output $\mathbf{y}_{1}$ decreases. The properties of system (7) with $u_{2}$, introduced in Assumption 1, ensure the desired boundedness of the output $\mathbf{y}_{2}$.

In Theorem 1 it is required that the closed-loop system satisfy (5) $-c f$. (13) and, as opposed to (4), the weaker estimates (11) and (12). The following Corollary establishes an exponential bound by imposing, besides the latter estimates, a specific rate of convergence.

Corollary 1. Let all conditions of Theorem 1 hold, $T_{1}(s) \leq \tau_{1}$ for $0 \leq s \leq R_{3}, s_{1}, s_{2}, s_{3}, s_{4} \in R_{+}, \sigma_{1}, \sigma_{2}, \sigma_{3}, \sigma_{4} \in K$ and

$$
\omega_{2}\left(s_{1}, s_{2}, s_{3}, s_{4}\right) \leq \sigma_{2}\left(s_{2}\right)\left(\sigma_{1}\left(s_{1}\right)+\sigma_{3}\left(s_{3}\right)+\sigma_{4}\left(s_{4}\right)\right),
$$

Then, for all initial conditions $\mathbf{x}_{0} \in X, \mathbf{d} \in \Omega$ and $t \geq t_{0} \geq 0$

$$
\begin{array}{r}
\left|\mathbf{y}_{1}(t)\right| \leq \tilde{\beta}\left(\lambda^{-1}\left|\mathbf{y}_{1}\left(t_{0}\right)\right| \mathrm{e}^{\frac{\ln (\lambda)\left[t-t_{0}\right]}{\tau_{1}+\tau_{D}}}\right)+\tilde{\gamma}\left(S\left[\mathbf{d}, t_{0}, t\right]\right), \\
\tilde{\beta}(s)=\max \left\{\beta_{1}(2 s, 0), \sigma_{2}\left(\max \left\{\Delta, R_{2}\right\}\right) \sigma_{1}(2 s)\right\}, \\
\tilde{\gamma}(s)=\max \left\{\sigma_{2}\left(\max \left\{\Delta, R_{2}\right\}\right)\left[\sigma_{1}(2 \Lambda \gamma(s))+\sigma_{3}(\gamma(s))\right],\right. \\
\left.\beta_{1}(2 \Lambda \gamma(s), 0)+\gamma(s)\right\} .
\end{array}
$$

Condition (14) implies (4) and under conditions of the corollary the switched system (3), (8), (9) admits all desired properties. The main disadvantage of Theorem 1 and the Corollary 1 relies in the complexity of the conditions. When no disturbances are considered we can simplify the conditions in following way.

Theorem 2 (without disturbances). Let Assumption 1 hold, $\mathbf{d}(t) \equiv 0, t \geq 0$ and $X=\left\{\mathbf{x} \in R^{n}:\left|\mathbf{h}_{1}(\mathbf{x})\right| \leq R_{1}\right\}, R_{1} \geq$ $0, R_{2}=\rho\left(R_{1}\right)+\rho_{0}$. Assume that there exist $\Delta$ and $\delta$ satisfying $\Delta>\max \left\{\delta, \rho_{0}, \omega_{1}(s, \delta, D, 0)\right\}$ and let $T_{1}: R_{+} \rightarrow R_{+}$be the solution of $\Delta=\omega_{1}\left(s, \delta, 0, T_{1}(s)\right), s \geq 0$ and $\tau_{D} \geq 0$ satisfy $\beta_{2}\left(\Delta, \tau_{D}\right)=\delta$. Furthermore, let

$$
\beta_{1}\left(s, T_{1}(s)\right)<s, \quad 0<s \leq R_{3},
$$

$R_{3}=\max \left\{\beta_{1}\left(R_{1}, 0\right)+\gamma(D), \omega_{2}\left(R_{1}, \max \left\{\Delta, R_{2}\right\}, 0,0\right)\right\} ;$

$$
i(t)=2, \quad t \in\left[t_{1}, t_{2}\right) \quad \Rightarrow \quad\left|\mathbf{y}_{1}\left(t_{2}\right)\right| \leq\left|\mathbf{y}_{1}\left(t_{1}\right)\right|
$$


Then, the system (3), (8), (9) is forward complete and for all initial conditions $\mathbf{x}_{0} \in X$ and $t \geq t_{0} \geq 0$ it holds that:

$$
\begin{aligned}
\left|\mathbf{y}_{1}(t)\right| \leq \max \{ & \beta_{1}\left(\left|\mathbf{y}_{1}\left(t_{0}\right)\right|, 0\right), \\
\left.\omega_{2}\left(\left|\mathbf{y}_{1}\left(t_{0}\right)\right|, \max \left\{\Delta,\left|\mathbf{y}_{2}\left(t_{0}\right)\right|\right\}, 0,0\right)\right\}, & \\
& \lim _{t \rightarrow+\infty}\left|\mathbf{y}_{1}(t)\right|=0 \\
\left|\mathbf{y}_{2}(t)\right| & \leq \beta_{2}\left(\max \left\{\Delta,\left|\mathbf{y}_{2}\left(t_{0}\right)\right|\right\}, 0\right)
\end{aligned}
$$

Condition (15) means that the the output trajectories of (7) under control $u_{1}$ i.e., $\mathbf{y}_{1}$, is strictly decreasing while during activity of control $u_{2}$ the output $\mathbf{y}_{1}$ does not increase and the output $\mathbf{y}_{2}$ passes from level $\Delta$ to $\delta$. In this case the supervisor (9) provides for the system (3) with uniting control (8) the desired boundedness and convergence of the output $\mathbf{y}_{1}$ to zero with estimate (5) for the output $\mathbf{y}_{2}$.

\section{A. Example: control of a unicycle with collision avoidance}

Consider the unicycle model:

$$
\begin{aligned}
& \dot{x}=\cos (\theta) u_{1} \\
& \dot{y}=\sin (\theta) u_{1} \\
& \dot{\theta}=u_{2}
\end{aligned}
$$

where $x \in R, y \in R$ are Cartesian coordinates on the plane, $\theta \in R$ is the angle of the driving-wheel, $u_{1} \in R$ and $u_{2} \in R$ are controls and $u=\left(u_{1}, u_{2}\right)$. It is required to drive the robot from any position to the origin on the plane (i.e., to stabilize the point $x=y=0$ ). Additionally, it is assumed that there are obstacles on the plane, which must be avoided during maneuvers. The obstacles are defined as follows: for a given set $Z=\left\{\left(\xi_{i}, \psi_{i}, \rho_{i}\right)\right\}_{i=\overline{1, N}}$ the pairs $\left(\xi_{i}, \psi_{i}\right)$ define the coordinates of the obstacles' centers on the plane and $\rho_{i}>0$ denote safety distances to the centers which must be maintained during the robot's motion. $N>0$ is the number of obstacles. We assume that the robot always starts at an initial position far from the obstacles and that for any $1 \leq i \leq N, 1 \leq j \leq N, i \neq j$ there exist $R_{i}>\rho_{i}, R_{j}>\rho_{j}$ such that $\sqrt{\left(\xi_{i}-\xi_{j}\right)^{2}+\left(\psi_{i}-\psi_{j}\right)^{2}}>$ $R_{i}+R_{j}$. The last condition means that between any obstacles there always exists a space where the robot can pass through.

Based on the previous set-up we choose the outputs to be regulated as:

$$
\begin{gathered}
y_{1}=\sqrt{x^{2}+y^{2}}, \quad y_{2}=\operatorname{dist}_{Z}(x, y)^{-1}, \\
\operatorname{dist}_{Z}(x, y)=\min _{1 \leq i \leq N}\left\{\sqrt{\left(x-\xi_{i}\right)^{2}+\left(y-\psi_{i}\right)^{2}}\right\} .
\end{gathered}
$$

The first output is the distance to the origin on the plane, the second output is the inverse of the distance to the set $Z$. In this case, if output $y_{2}$ goes to zero the distance to the set $Z$ goes to infinity hence, preserving the values of output $y_{2}$ sufficiently small we can ensure collision avoidance.

In accordance with the proposed approach it is necessary to design two controls which provide independent stabilization of the outputs $y_{1}$ and $y_{2}$ in the sense of Assumption 1. Then, the supervisor (9) ensures the desired position stabilization with collisions avoidance. As is well-known the unicycle is not stabilizable via smooth stationary feedback; here, we use right-continuous controls as imposed by the main results.

Control Task 1: stabilization. This task consists in steering the unicycle to the origin of the plane i.e., in stabilizing $y_{1}=$ 0 . Consider the Lyapunov function $V_{1}\left(y_{1}\right)=0.5 y_{1}^{2}$ whose time-derivative is $\dot{V}_{1}=(x \cos (\theta)+y \sin (\theta)) u_{1}$. Let $\theta_{0}=$ $\operatorname{atan}\left(y x^{-1}\right)$ be the angle between a line connecting the origin to the point $(x, y)$ on the plane and the horizontal axis. Then, $x y_{1}^{-1}=\cos \left(\theta_{0}\right), y y_{1}^{-1}=\sin \left(\theta_{0}\right)$. Define $\theta=\theta_{0}+\pi+\alpha$, where $\theta_{0}+\pi$ is the desired unicycle orientation, $\theta$ and $\alpha \in$ $[-\pi, \pi]$ is the deviation from the reference $\theta_{0}+\pi$. Then, $\dot{V}_{1}=$ $\left(\cos \left(\theta_{0}\right) \cos (\theta)+\sin \left(\theta_{0}\right) \sin (\theta)\right) y_{1} u_{1}=\cos \left(\theta_{0}-\theta\right) y_{1} u_{1}=$ $-\cos (\alpha) y_{1} u_{1}$. Hence,

$$
u_{1}=\left\{\begin{array}{l}
y_{1} \text { if }|\alpha| \leq \kappa \pi \\
0 \text { if }|\alpha|>\kappa \pi
\end{array}\right.
$$

with $0<\kappa<0.5$ ensures negative semi-definiteness of $\dot{V}$. Since $\dot{\alpha}=u_{2}+\sin (\alpha) y_{1}^{-1} u_{1}$, by substituting the control

$$
u_{2}=-k_{1} \alpha-\left\{\begin{array}{l}
\sin (\alpha) \text { if }|\alpha| \leq \kappa \pi \\
0 \text { if }|\alpha|>\kappa \pi
\end{array}\right.
$$

we obtain $\dot{\alpha}=-k_{1} \alpha$ which implies exponential decreasing for the deviation variable $\alpha(t)=\alpha(0) \exp \left(-k_{1} t\right)$. For such $\alpha(t)$ both proposed controls have only one jump hence, are rightcontinuous as required. Note that for $|\alpha|>\kappa \pi$ we have $\dot{V}_{1}=0$, while for $|\alpha| \leq \kappa \pi$

$$
\dot{V}_{1} \leq-c_{\min } y_{1}^{2}=-2 c_{\min } V_{1}, \quad \mathrm{c}_{\min }=\min _{|\alpha| \leq \kappa \pi}\{\cos (\alpha)\}>0
$$

which also implies that $\left|y_{1}(t)\right| \rightarrow 0$ exponentially. The time interval when $|\alpha|>\kappa \pi$ is bounded by $T_{\max }=\frac{1}{k_{1}} \ln \left(\frac{1}{\kappa}\right)$. This is the time required for the controller $u_{2}$, to make the robot turn from angle $\pm \pi$ to $\pm \kappa \pi)$. Then, the variable $y_{1}$ satisfies the exponential estimate: $y_{1}(t) \leq y_{1}(0) \exp \left(-c_{\min }\left[t-T_{\max }\right]\right)$ for all $t \geq T_{\max }$.

Task 2: Obstacle avoidance. We design now the controller $u_{2}$. To that end, we assume without loss of generality (since between any obstacles there is a minimal distance) that the control goal is to avoid the obstacle parameterized by $\left(\xi_{i}, \psi_{i}, \rho_{i}\right)$. The control $u_{2}$ must be such that the distance from level $\rho_{i}$ (when control is switched on) up to the level $R_{i}>\rho_{i}$ increases $-c f$. Fig. 1. Let $\Delta_{i}=\rho_{i}^{-1}$ and $\delta_{i}=R_{i}^{-1}$ then, control $\mathbf{u}_{2}$ must ensure that the output $y_{2}$ decreases from $\Delta_{i}$ to $\delta_{i}$. An additional requirement is that the distance $y_{1}$ does not increase; this restricts the set of possible directions for the angle $\theta$.

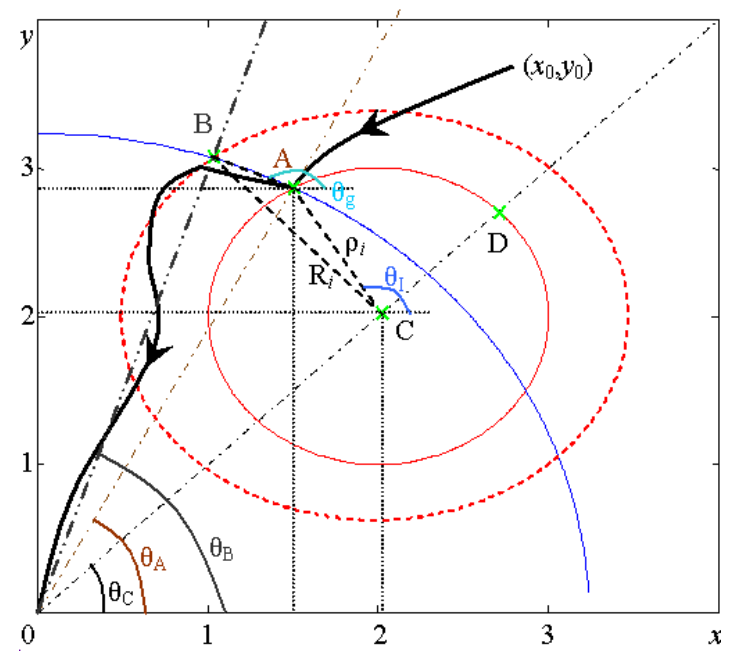

Fig. 1. Illustration of possible path for collision avoidance 
For illustration, consider an obstacle within a circle centered at a point $\mathrm{C}$ and a trajectory with initial conditions $\left(x_{0}, y_{0}\right)$ at point $\mathrm{A}$ which is the point of intersection of the trajectory and the circle centered at point $\mathrm{C}$ and of radius $\rho_{i}$. The control $u_{2}$ which becomes active when the robot is located at point $\mathrm{A}$, has to push the trajectory outside the circle with radius $R_{i}$ but not exceeding point $\mathrm{B}$ in module i.e., the actual robot path must remain below the arc centered at the origin and with radius, the module of point $\mathrm{B}^{3}$. Let $\left(x_{A}, y_{A}\right)$ be the coordinates of the point $\mathrm{A}$ relative to point $\mathrm{B}$ are

$$
\begin{gathered}
x_{B}=\frac{1}{2}\left(\xi_{i}-\frac{\xi_{i}\left(R_{i}^{2}-R_{A}^{2}\right) \pm \Upsilon}{\xi_{i}^{2}+\psi_{i}^{2}}\right) ; \\
\Upsilon:=\psi_{i} \sqrt{\left(\left[R_{i}+R_{A}\right]^{2}-\xi_{i}^{2}-\psi_{i}^{2}\right)\left(\xi_{i}^{2}+\psi_{i}^{2}-\left[R_{i}-R_{A}\right]^{2}\right)} \\
y_{B}= \pm \sqrt{R_{A}^{2}-x_{B}^{2}} ; \quad R_{A}=\sqrt{x_{A}^{2}+y_{A}^{2}} .
\end{gathered}
$$

The sign of the square root in $y_{B}$ should be chosen such that the following property is satisfied:

$$
\operatorname{sign}\left(\theta_{C}-\theta_{A}\right)=\operatorname{sign}\left(\theta_{C}-\theta_{B}\right),
$$

where $\theta_{C}, \theta_{A}$ and $\theta_{B}$ are corresponding angles between the horizontal axis and the lines to points $\mathrm{C}, \mathrm{A}, \mathrm{B}$ shown in Fig. 1. Roughly, the last equality ensures that the robot is steered by the controls away from the obstacle. The so-calculated point $\mathrm{B}$ indicates the direction such that the distance to the obstacle increases while not increasing the distance to the origin. See Fig. 1 .

Let us now consider the Lyapunov function

$$
V_{2}(x, y)=0.5 y_{2}^{2}=0.5\left[\left(x-\xi_{i}\right)^{2}+\left(y-\psi_{i}\right)^{2}\right]^{-1},
$$

whose time derivative is

$$
\begin{aligned}
\dot{V}_{2} & =-\frac{\left(x-\xi_{i}\right) \cos (\theta)+\left(y-\psi_{i}\right) \sin (\theta)}{\left[\left(x-\xi_{i}\right)^{2}+\left(y-\psi_{i}\right)^{2}\right]^{2}} u_{1} \\
& =-\cos \left(\theta_{I}-\theta\right) y_{2}^{3} u_{1},
\end{aligned}
$$

where angle $\theta_{I}$ is shown in Fig. 1, it defines the orthogonal direction from the center of the obstacle (movement in this direction will increase the distance to the obstacle in the fastest way). The goal direction, which determines the point $\mathrm{B}$ can be defined by the angle $\theta_{g}=\operatorname{atan}\left(\left(y_{B}-y\right)\left(x_{B}-x\right)^{-1}\right)$, which is also shown in Fig. 1. Let $\theta=\theta_{g}-\alpha$, where $\alpha \in[-\pi, \pi]$ is the deviation from the desired angle $\theta_{g}$, then $\dot{V}_{2}=-\cos \left(\theta_{I}-\theta_{g}+\alpha\right) y_{2}^{3} u_{1}$. The variable $\alpha$ obeys the following differential equation:

$$
\dot{\alpha}=\dot{\theta}_{g}-\dot{\theta}=\sin (\alpha)\left[\left(x_{B}-x\right)^{2}+\left(y_{B}-y\right)^{2}\right]^{-0.5} u_{1}-u_{2}
$$

If $\left|\theta_{I}-\theta_{g}\right|<0.5 \pi$, then there exists $0.5>\varepsilon>0$ such that

$$
\begin{aligned}
& \text { controls } \\
& \qquad u_{1}= \begin{cases}y_{2}^{-1} \text { if }|\alpha| \leq \varepsilon \pi ; \\
0 \text { if }|\alpha|>\varepsilon \pi,\end{cases} \\
& u_{2}=k_{2} \alpha+ \begin{cases}\sin (\alpha) \sqrt{\left(x_{B}-x\right)^{2}+\left(y_{B}-y\right)^{2}} y_{2}^{-1} & \text { if }|\alpha| \leq \varepsilon \pi \\
0 & \text { if }|\alpha|>\varepsilon \pi\end{cases}
\end{aligned}
$$

ensure that $\dot{V}_{2} \leq 0$ and exponential convergence to zero of error variable $\alpha, k_{2}>0$. Indeed, in this case $\dot{\alpha}=-k_{2} \alpha$ and $\alpha(t)=$ $\alpha(0) \exp \left(-k_{2} t\right)$, for $|\alpha| \leq \varepsilon \pi$ the inequality $\dot{V}_{2} \leq-2 d_{\min } V_{2}$ holds, where $d_{\text {min }}=\min _{|\alpha| \leq \varepsilon \pi}\left\{\cos \left(\theta_{I}-\theta_{g}+\alpha\right)\right\}>0$. For the case $|\alpha|>\varepsilon \pi$ we have $\dot{V}_{2}=0$. Thus the distance to the obstacle

\footnotetext{
${ }^{3}$ In case $\mathrm{A}$ coincides with $\mathrm{D}$, the point $\mathrm{B}$ can be determined arbitrarily to the right or left of segment $(\mathrm{O}, \mathrm{C}, \mathrm{D})$.
}

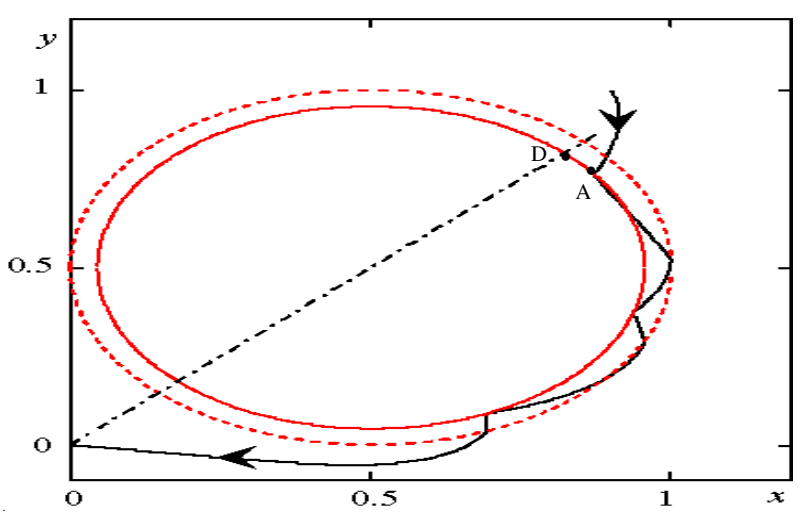

is not decreasing and the estimate $y_{2}(t) \leq y_{2}(0) \exp \left(-d_{\min }[t-\right.$ $\left.T_{\max }^{\prime}\right]$ ) for all $t \geq T_{\max }^{\prime}$ holds, where $T_{\max }^{\prime}=\frac{1}{k_{1}} \ln \left(\frac{1}{\kappa}\right)$ is the maximal amount of time during which the robot is steered towards the point B (away from the obstacle).

In the previous computations we have implicitly assumed that $\left|\theta_{I}-\theta_{g}\right|<0.5 \pi$; if this does not hold (for instance, if the point A lies "too" close to point $\mathrm{D}$ ) then, the distance to the obstacle can decrease proportionally to $\varepsilon, \rho_{i}$ and $R_{i}$, entailing that the unicycle enters the obstacle boundary. However, there always exists $\chi>1$ such that $y_{2}(t) \leq \chi y_{2}(0) \exp \left(-d_{\min }\left[t-T_{\max }^{\prime}\right]\right)$. We conclude that the system with controllers $\mathbf{u}_{1}$ and $\mathbf{u}_{2}$ satisfy the conditions of Assumption 1 hold and, in the absence of input disturbances, we can apply the result of the Theorem 2 .

For the purpose of simulation, we used $N=1, \xi_{1}=\psi_{1}=0.5$, $\rho_{1}=0.5, R_{1}=0.55, k_{1}=k_{2}=5, \kappa=1 / 3, \varepsilon=1 / 24$. The phase curves of the unicycle converging to the origin with a constrained motion are depicted in Fig. 2.

\section{CONCLUSION}

We have posed a problem of output regulation with respect to two outputs. The proposed solution consists in a supervisor that ensures input-output stability in the sense that one output is driven to zero (resp. a small compact in the presence of disturbances) and another is kept within a pre-specified bound. The second output is used to generate the switching signal that defines the supervisor. Typically, motivated by technical obstructions, in switched or supervisory systems theory a single output is used for switching; this has been overcome in this paper. A simple amid motivating example is presented: switched output regulation of the unicycle ensuring obstacle avoidance.

\section{REFERENCES}

[1] D. Liberzon, Switching in Systems and Control. Systems and Control: Foundations and Applications, Boston, MA: Birkhäuser, 2003. ISBN 0-8176-4297-8.

[2] A. S. Morse, "Supervisory control of families of linear set-point controllers-Part I: exact matching," IEEE Trans. on Automat. Contr., vol. 41, pp. 1413-1431, 1996.

[3] A. Teel and N. Kapoor, "Uniting local and global controllers," in Proc. 4th. European Contr. Conf., (Brussels, Belgium), 1997. Paper no. 959.

[4] P. Morin, R. Murray, and L. Praly, "Nonlinear rescaling of control laws with application to stabilization in the presence of magnitude saturation," in Proc. IFAC NOLCOS, (Enschede, The Netherlads), 1998. 
[5] C. Prieur, "Uniting local and global controllers with robustness to vanishing noise," Math. of Cont. Sign. and Syst., vol. 14, pp. 143172, 2001.

[6] C. Prieur and L. Praly, "Uniting local and global controllers," in Proc. 38th. IEEE Conf. Decision Contr., (Phoenix, AZ), pp. 12141219, 1999.

[7] C. Prieur and A. Astolfi, "Robust stabilization of chained systems via hybrid control," IEEE Trans. on Automat. Contr., vol. 48, no. 10, pp. 1768-1772, 2003.

[8] H. Ito and R. Freeman, "Uniting local andglobal controllers for uncertain nonlinear systems: beyond global inverse optimality," Syst. \& Contr. Letters, vol. 45, pp. 59-79, 2002.

[9] D. V. Efimov, "Uniting global and local controllers under acting disturbances," Automatica, vol. 42, no. 6, pp. 549-554, 2006.

[10] D. V. Efimov and A. L. Fradkov, "Finite time practical stabilization of nonlinear detectable systems by uniting control," in Proc. 46th. IEEE Conf. Decision Contr., (New Orleans, LA, USA), 2007.

[11] A. S. Morse, Trends in control, ch. Control using logic-based switching, pp. 69-113. Springer-Verlag, 1995. A. Isidory, ed.

[12] L. Vu, D. Chatterjee, and D. Liberzon, "Iss of switched systems and applications to switching adaptive control," in Proc. 44th. IEEE Conf. Decision Contr., pp. 120-125, 2005.

[13] W. Xie, C. Wen, and Z. Li, "Input-to-state stabilization of switched nonlinear systems," IEEE Trans. on Automat. Contr., vol. 46, pp. 1111-1116, 2001.

[14] P. Mhaskara, N. H. El-Farrab, and P. D. Christofides, "Stabilization of nonlinear systems with state and control constraints using lyapunov-based predictive control," Syst. \& Contr. Letters, no. 55, pp. 650-659, 2006.

[15] J. Hespanha, D. Liberzon, and A. S. Morse, "Hysteresis-based switching algorithms for supervisory control of uncertain systems," Automatica, vol. 39, pp. 263-272, 2003.

[16] J. Hespanha and A. Morse, "Stability of switched systems with average dwell-time," in Proc. 38th. IEEE Conf. Decision Contr. (Phoenix, AZ), pp. 2655-2660, 1999.

[17] E. D. Sontag and Y. Wang, "Notions of Input-to-Output stability," Syst. \& Contr. Letters, vol. 38, pp. 235-248, 1999. 